Artículo de reflexión

Apuntes del CENES

ISSN 0120-3053

Volumen $33-\mathrm{N}^{\circ} .58$

Julio - Diciembre de 2014

Págs. 217-242

\title{
Las competencias emprendedoras en el departamento de Boyacá
}

\author{
Entrepreneurial skills in Boyacá
}

Diana Cristina Rodríguez Moreno*

Adriana Ximena Gómez Murillo**

Fecha de recepción: 1 de abril de 2014

Concepto de evaluación: 6 de mayo de 2014

Fecha de aprobación: 15 de septiembre de 2014

Administradora de empresas. Magíster en Administración. Profesora titular de la Escuela de Administración de Empresas, Universidad Pedagógica y Tecnológica de Colombia. Tunja, Colombia. Correo electrónico: diana. rodriguez@uptc.edu.co

** Administradora de empresas. Universidad Pedagógica y Tecnológica de Colombia. Tunja, Colombia. Correo electrónico: adriana-xgm581@hotmail.com 


\title{
Resumen
}

Este trabajo presenta algunas reflexiones acerca de las competencias emprendedoras en el departamento de Boyacá, a partir de los resultados obtenidos en una exploración realizada en el año 2013, durante el desarrollo del segundo concurso regional de emprendimiento, cuyo proceso y resultados también son expuestos. Para este trabajo, fueron seleccionadas algunas competencias y se aplicaron instrumentos utilizando la escala Likert que permitieron establecer el nivel de competencias de emprendedores y potenciales en prendedores de Boyacá. Los emprendedores boyacenses confían en su capacidad para el logro de objetivos, baja capacidad para trabajo en equipo, individualismo y liderazgo débil.

Palabras clave: competencias emprendedoras, emprendedores, concurso emprendimiento.

JEL M 13, L 26, M 19.

\begin{abstract}
This paper presents some reflections about entrepreneurial skills in the department of Boyacá, from the results obtained in 2013, during the development of the second regional entrepreneurship contest, whose process and results are also presented. For this work were selected some entrepreneur skills. Were applied tools using Likert scale for drawing up the skill level of entrepreneurs in Boyacá. The entrepreneurs have ability to achieve goals, low capacity for teamwork and weak leadership.
\end{abstract}

Keywords: entrepreneurial skills, entrepreneur, entrepreneur contest. 


\section{INTRODUCCIÓN}

Hoy existe una marcada tendencia en todos los países del mundo, hacia la promoción del emprendimiento desde las políticas gubernamentales. En los ámbitos nacional y regional (O'Connor, 2013) se ha establecido que el desarrollo del emprendimiento constituye el camino hacia el progreso y es importante su fomento, pues favorece todo tipo de organizaciones tanto públicas como privas, además empresas grandes medianas y pequeñas, y también genera desarrollo en los mercados nacional e internacional. (Soriano \& Huarng, 2013). Con este argumento, entre otros, los gobiernos nacionales, departamentales y municipales, así como las universidades $y$ en general centros educativos se han puesto a la tarea de generar estrategias que fomenten el emprendimiento. En este sentido, Drucker presenta la necesidad de una sociedad emprendedora, así la innovación y el emprendimiento deben estar presentes en las empresas, la economía y la sociedad (Heinonen \& Ruotsalainen, 2012). Es ampliamente aceptado que la sociedad capitalista requiere del emprendimiento para crecer y progresar (Birds, 2014), el emprendimiento es crítico para el desarrollo económico (Stuetzer, Obschonka, y Schmitt-rodermund, 2013, Stuetzer, Goethner y Cantner, 2012). Adicionalmente es un área de estudio muy útil para individuos, sociedades y países (Matiz, 2009).

Este trabajo presenta la experiencia obtenida en la investigación liderada por los grupos de investigación Competitividad y Desarrollo Local (CODEL) y Gerencia del Valor y Finanzas (GEVAFI) de la Escuela de 
Administración de Empresas de la Universidad Pedagógica y Tecnológica de Colombia, en articulación con la Gobernación de Boyacá, Secretaria de Productividad, Tic y Gestión del Conocimiento y el apoyo de la Red Regional de Emprendimiento del departamento de Boyacá. En este proyecto se plantearon dos objetivos: el primero, realizar un diagnóstico de algunas competencias emprendedoras de los emprendedores boyacenses y su nivel de conocimientos administrativos básicos en fianzas y mercados, y el segundo, desarrollar el segundo concurso departamental de emprendedores.

En la primera sección se presenta una explicación del surgimiento del proyecto, con sus actores; en la segunda sección se expone el proceso de determinación de competencias emprendedoras, y una tabla con las competencias, su definición y las conclusiones obtenidas del estudio; en el tercer apartado se muestra el desarrollo del mencionado concurso, sus etapas y selección de ganadores. Finalmente se hacen algunas reflexiones a manera de conclusiones.

\section{ACTORES Y SURGIMIENTO DEL PROYECTO}

Hacia el primer semestre de 2012, la Secretaría de Productividad, TIC y Gestión del Conocimiento de la Gobernación de Boyacá expresó su interés en promocionar y fortalecer la cultura emprendedora en el departamento, contemplada en el proyecto "Implementación del programa para el fomento de la cultura del emprendimiento en el departamento de Boyacá" (Estudios Previos Convenio de Cooperación Interadministrativo, 2012) donde la estrategia de articulación institucional como herramienta para el fomento de la cultura del emprendimiento permitió la suscripción de un convenio de cooperación interadministrativo con la Universidad Pedagógica y Tecnológica de Colombia (Uptc) para fortalecer las competencias emprendedoras y empresariales del departamento de Boyacá apoyado por la Red Regional de Emprendimiento.

El objetivo central del proyecto es el fomento de la actividad emprendedora en el departamento, con la realización de eventos de sensibilización en competencias emprendedoras; evaluar algunas competencias emprendedoras en el departamento, diseñar los criterios y la metodología para determinar las mejores ideas y modelos de negocio y desarrollar el segundo concurso departamental de emprendedores, como estímulo a los emprendedores o empresarios que voluntariamente decidieron participar en el proceso.

La propuesta fue apoyada por la Red Regional de Emprendimiento, equipo conformado por actores del sector gubernamental, empresarial y académico interesados en el fomento de la cultura del emprendimiento en el departamento. Este apoyo fue un factor indispensable de motivación para el proceso. También se buscó el apoyo de las cámaras de 
comercio de Tunja, Duitama y Sogamoso, con las bases de datos de empresarios, para realizar la promoción de la propuesta, el apoyo logístico en espacios físicos para el desarrollo de los talleres de sensibilización y demás eventos. También se contó con la participación del Fondo Regional de Garantías como garante del proceso del concurso.

\section{DETERMINACIÓN DE COMPE- TENCIAS EMPRENDEDORAS}

Para conocer el perfil del emprendedor boyacense se utilizó el enfoque de competencias. En este apartado se hace una breve referencia al emprendimiento y a las competencias, y en último término se determinan las competencias emprendedoras que fueron evaluadas.

Emprendimiento. El término emprendimiento se ha relacionado especialmente con innovación, aunque también con empresa, cambio, empleo, valor y crecimiento (Torres, 2010). Schumpeter es considerado un pionero en el análisis económico de la iniciativa empresarial (Karp, 2006) por su contribución significativa a la teoría del emprendimiento. Para este autor, el espíritu empresarial es el motor principal del desarrollo económico con la innovación como elemento central (Lordkipanize, Bretzel \& Backman, 2005). La función del emprendedor es explotar un invento o una posibilidad tecnológica que genere nuevos productos o servicios, nuevas formas de producción, fuentes de suministro de materias primas, formas de organización que revolucionan el patrón de producción establecido (Shumpeter, 1943). Un emprendedor es alguien que identifica una necesidad en el mercado, toma decisiones sobre recursos humanos, financieros, materiales, también toma riesgos, lo cual es recompensado por beneficios económicos (Ras \& Vermeulen, 2009). El emprendedor asume riesgos financieros, sociales, sociológicos, invierte tiempo y esfuerzo para recibir una recompensa monetaria y de satisfacción personal (Antonia, Ciencias, Ve, Zulia \& Cervilla, 2013, citando a Hisrish, 2006)

Hay seis escuelas de pensamiento que definen el espíritu empresarial: la escuela del emprendimiento de "la gran persona" y la de "características psicológicas" que precisan al emprendedor de acuerdo con sus cualidades personales; la escuela clásica, que define al emprendedor como aquel que tiene la habilidad de reconocer oportunidades; la escuela de la gerencia y la del liderazgo, que se centran en las habilidades de gestión de los empresarios, y la sexta escuela, que define el emprendimiento en la organización o en el interior de esta (intrapreneurs) como la capacidad de adaptarse. (Cunningham y Lischeron, 1991, citado en Sambasivan et al., 2009)

\section{Competencias}

Según la Real Academia Española, competencia en segundo significado es "pericia, aptitud, idoneidad para hacer algo o intervenir en un asunto determinado". La competencia combina recursos como conocimientos, saber hacer y aptitudes 
(Higuita, Molano \& Rodríguez, 2011). Existe relación entre competencias, aptitudes y rasgos de personalidad, aunque competencia se refiere también a conocimiento adquirido por un individuo a través de la experiencia, por eso están vinculadas a la práctica de una actividad específica. Las competencias se materializan en comportamientos que se llevan a cabo cuando se ponen en práctica los conocimientos, aptitudes y rasgos de personalidad (Martínez \& Carmona, 2009). Entonces, una característica esencial de la competencia es la acción. Así, una competencia se edifica integrando motivación, conocimiento, valores, actitudes y emociones, para generar una acción (Fernández \& Fernandez, 2010).

Dentro de las teorías socioculturales de la personalidad, la de los rasgos se centra en determinar cuáles son los rasgos de personalidad que debería tener un individuo para realizar determinados objetivos u ocupar un cargo. A manera de ejemplo, una persona puede ser más o menos extrovertida, más o menos segura, sumisa o dominante, etc., sin embargo, los rasgos de personalidad no permiten predecir el comportamiento de los individuos en el trabajo, es más, un determinado rasgo puede ser percibido positiva o negativamente por distintas personas o dependiendo de contextos específicos, esta es una de las razones por las cuales el enfoque de competencias facilita la comprensión de esas habilidades e intenciones de las personas que se verifican a través de sus comportamientos.
El enfoque de competencias se centra en comportamientos observables, en este sentido, se orienta más a los resultados que se esperan de la persona. No es suficiente tener el conocimiento, pues los resultados de una persona pueden no ser consecuentes con sus títulos y experiencia, en otras palabras, las acciones de las personas pueden distar de lo que se sabe o lo que se dice. Es importante no solo saber, es indispensable saber hacer, poder hacer y querer hacer. La administración moderna combina gestión de resultados con gestión de competencias. El individuo logra un desempeño adecuado mediante la aplicación de conocimientos, habilidades, experiencias, aptitudes y motivaciones, en conductas que le permiten resolver problemas o enfrentar situaciones propias de su entorno (Castellanos, 2006).

Mertens (2000) define competencia como una aptitud que se logra con la adquisición y desarrollo de conocimientos, habilidades y capacidades que son expresadas en el saber, el hacer y el saber hacer. Son un conjunto de propiedades en permanente modificación, sometidas a la prueba de resolución de problemas concretos que presentan cierto grado de incertidumbre y complejidad técnica (Gallard \& Jacinto, 1995).

\section{Competencias emprendedoras}

El emprendimiento, de manera funcional, se asume como una actitud (Castellanos, Chávez \& Jiménez, 2003). El emprendimiento requiere de competencias o, dicho de otro modo, las personas que 
emprenden una idea de negocio tienen ciertas competencias. El emprendedor se moviliza por deseo, por necesidad u oportunidad (Rogoff, 2007, citado en Torres, 2010)

- Así, las competencias emprendedoras implican capacidades, destrezas, habilidades y aptitudes que hacen posible que el emprendedor se desempeñe de manera idónea en el proceso emprendedor (Gómez \& Zatizábal, 2011). En este enfoque basado en el comportamiento, la acción de emprender es un proceso que necesariamente lleva al desarrollo de una idea con la creación de una empresa (Alda, Villardón \& Elexpuru, 2012). También permite determinar si una persona puede hacer realidad su intención de poner en marcha una nueva empresa y hacerla crecer de manera exitosa, entre mayor habilidad tiene el emprendedor, más se beneficia del negocio (PlehnDujowich, 2009).

El emprendedor tiene capacidad de generar ideas, convertirlas en algo novedoso para transformar positivamente su vida y su entorno (Duarte \& Ruiz, 2009). El emprendedor genera motivación en otros para lograr un objetivo. Por otra parte, las decisiones de los emprendedores se ven influenciadas por las opiniones de otros, incluso la decisión de participar en una iniciativa empresarial (Bosma et al., 2012), así el emprendimiento va más allá de lo individual, además implica la interacción con otros, con el entorno y está inmerso en una cultura específica (Orrego, 2008). Entonces el estudio del emprendimiento comprende atributos económicos, psicológicos, sociales y culturales (Rodriguez, 2009).

La existencia de una oportunidad empresarial no es suficiente para garantizar el espíritu empresarial, además es necesario contar con la capacidad para tomar decisiones en asignación de recursos y explotación de los mismos para obtener beneficio empresarial (D'Este, Mahdi, Neely \& Rentocchini, 2012). La cultura nacional influencia el emprendimiento (Zapalska, Dabb \& Perry, 2003). El rendimiento de nuevas empresas depende de la capacidad del emprendedor de influir en las acciones de stakeholders, así la competencia social del empresario tiene gran importancia y dentro de esta la habilidad política que consiste en la capacidad del individuo para entender a otros y utilizar ese conocimiento para influir en los demás (Tocher, Oswald \& Shook, 2010)

La literatura acerca del potencial emprendedor es difusa, no hay una definición consensuada tampoco una conceptualización de la manifestación potencial empresarial y su medición (Santos, Caetano, \& Curral, 2013). Santos et al., (2013), citando a Raab, Stedham y Neuner (2005), definen el potencial emprendedor como el grado en que un individuo posee características que se asocian a los empresarios de éxito.

El proceso de la iniciativa empresarial está profundamente ligado a las características de un individuo que es el agente principal en la decisión de iniciativas empresariales 
y quien asume la responsabilidad y consecuencias, aunque se debe reconocer que la iniciativa empresarial no es únicamente el resultado de acciones y características de un individuo, pues los factores externos como el contexto económico, político, tecnológico y legal juegan un papel relevante (Santos et al., 2013). En este sentido otros autores sugieren que el espíritu empresarial no debería definirse únicamente en términos de que el empresario es y hace, sino analizando quién y con qué se crean bienes y servicios (Rhee \& White, 2007).

Pese a que la investigación sobre formación y desarrollo de capital humano empresarial es escasa, estudios empíricos reportan una baja correlación entre los indicadores de capital humano, como educación y experiencia, y el avance en la creación de empresa (Stuetzer, Obschonka, Davidsson, \& Schmitt-Rodermund, 2013). Se ha propuesto entonces un modelo teórico que da importancia a la combinación de aspectos de capital humano en habilidades o competencias equilibradas para empresarios (Lazear, 2005), pues deben poseer, combinar y equilibrar habilidades para el uso de diversos recursos como capital físico, capital humano y financiero (Stuetzer et al., 2012). Si el emprendedor cuenta con más habilidades, el costo de oportunidad de la coordinación de trabajadores, en términos productivos, es mayor, así trabajadores calificados deberían necesitar menos atención del empresario (Baptista, Lima \& Preto, 2012).
Birds (2014) estudia en una universidad de Inglaterra las competencias que debería tener el empresario universitario y específicamente se refiere a los profesores que eventualmente pueden ser gestores de spin off académicas. El emprendedor demuestra ciertas habilidades específicas, comportamientos y experiencia, presenta las diez competencias planteadas por Chakravarthy y Lorange (2008) y cada una permite ver el grado en que los individuos exhiben comportamientos empresariales en el curso de su empleo en la enseñanza superior. Las competencias son: pensamiento estratégico (ver el panorama general), capacidad de hacer marketing a la estrategia, capacidad para obtener apoyo y movilizar recursos, habilidades en la conformación y motivación de un equipo, asumir riesgos, orientación a la acción, confianza en sí mismo, experiencia y creación de redes, que consiste en el establecimiento de contactos y su utilización en beneficio del emprendimiento (Birds, 2014)

Santos, Caetano y Curral (2013) sostienen que cada individuo tiene un potencial para el emprendimiento, conformado por diversas competencias y motivaciones: las motivaciones emprendedoras, competencia en gerencia, competencias psicológicas y competencias sociales. Estas cuatro dimensiones incluyen once subdimensiones. Las motivaciones emprendedoras son el deseo de independencia, motivación económica, autoeficacia; las competencias gerenciales son visión, capacidad de movilización de recursos, capacidad de liderazgo; las capacidades 
psicológicas comprenden capacidad de innovación, inteligencia emocional, resiliencia (capacidad del individuo de superar una situación traumática) y finalmente las competencias sociales, como capacidad de comunicación y persuasión y capacidad de desarrollar redes.

Normalmente, los emprendedores fracasan una o varias veces en la búsqueda de realizar sus expectativas empresariales, algunos con el primer fracaso desisten, para otros esta no es una opción, pues su deseo es tan fuerte que siguen adelante con otras ideas, estos se llaman emprendedores en serie, que generan numerosas ideas y emprendimientos. Los emprendedores con altas habilidades, conservan su espíritu empresarial si su negocio no cumple con sus expectativas cierran y ponen en marcha uno nuevo, son emprendedores en serie, por otra parte los emprendedores con bajas habilidades, cierran su negocio y entran al mercado laboral o se mantienen en operación (Plehn-Dujowich, 2009)

Krueger y Brazeal ( 1994 ) presentan un modelo de potencial emprendedor, así una persona puede tener alto potencial emprendedor pero no necesariamente considera su participación en una actividad empresarial o viceversa (Santos et al., 2013). El potencial emprendedor es definido como un proceso de interacción entre el atractivo percibido, que tiene en cuenta las normas sociales y las actitudes; la viabilidad percibida que tiene que ver con la autoeficacia y la propensión a actuar (Santos et al., 2013).
Stedham y Neuner (2005) sugirieron que el potencial emprendedor está expresado por siete características: necesidad de logro, locus de control, la propensión a tomar riesgos, resolución de problemas, la voluntad de afirmarse, la tolerancia de la ambigüedad y la estabilidad emocional (Santos et al., 2013). Por otra parte, las competencias en emprendedoras futuras, teniendo en cuenta el auge tecnológico, son: competencias de colaboración, que se relacionan con las habilidades de trabajo en equipo, aprendizaje y creación conjuntos; competencias de tiempo, que se refieren al uso óptimo del tiempo; competencias tecnológicas; competencias medioambientales, que versan sobre la relación de las personas con la naturaleza y medio ambiente; competencia de sistemas, que corresponden al pensamiento holístico; competencias sociales, que tiene que ver con la habilidad de comprender diversos contextos culturales y estilos de vida (Heinonen \& Ruotsalainen, 2012).

El programa Bogotá Emprende define competencias emprendedoras como los conocimientos, habilidades, destrezas y actitudes para realizar el trabajo de emprendimiento, encierra una serie de atributos que comprenden elementos individuales y sociales y que se adquieren con la experiencia, así que exigen educación tanto formal (conocimiento) como no formal (vivencias, experiencia). Las competencias en emprendimiento propuestas por este programa, son capacidad de exploración (intención de incrementar conocimientos), pensamiento 
emprendedor (tener la iniciativa de actuar sobre el medio para modificarlo a través de la puesta en marcha de soluciones prácticas), pensamiento estratégico (tener claro un objetico, saber cómo alcanzarlo, realizar análisis estratégico para toma de decisiones), trabajo en equipo (trabajar en cooperación con otros para obtener un resultado articulado), liderazgo (consiste en influir en otros, implica procesos de comunicación, apuntando al logro de metas y objetivos), orientación a objetivos (enfocar el trabajo a alcanzar objetivos, teniendo en cuenta la relación costo-beneficio de las acciones), negociación (capacidad de llegar a acuerdos, solucionar conflictos y llegar a alianzas y negociaciones donde las partes resultan beneficiadas), proactividad (capacidad de anticiparse a necesidades, problemas y situaciones para proveer soluciones), proyección social (tener en cuenta el beneficio para otros, además del propio), habilidad personal (asertividad y claridad en la transmisión de información) e integridad personal (respeta principios, valores y personas) (Bogotá emprende, 2010).

En este trabajo se consideraron las competencias emprendedoras como aquellas necesarias para la creación de empresa, se indagó acerca de competencias personales basadas en probables compor- tamientos de emprendedores ante situaciones específicas. El enfoque basado en comportamientos se centra en el proceso emprendedor y en los comportamientos y actividades que en él se llevan a cabo. Una de las fortalezas de este enfoque radica en la conceptualización del fenómeno emprendedor, en donde el emprender es un proceso que concluye con la creación de una nueva empresa o el desarrollo de una idea.

Para las competencias se tuvo en cuenta el programa Bogotá Emprende, ya que ha sido el modelo más importante de aplicación en el país, y se tomaron seis de las once competencias (capacidad de exploración, orientación a objetivos, liderazgo, trabajo en equipo, proactividad y pensamiento estratégico), para cada una de las cuales fueron definidos cuestionamientos orientados a actitudes, utilizando la escala tipo Likert. El instrumento fue aplicado a emprendedores (156) de las principales ciudades del departamento (Tunja, Duitama y Sogamoso), quienes voluntariamente decidieron participar en el proceso y asistieron a tres eventos de sensibilización organizados para promocionar el segundo concurso departamental de emprendedores: uno en Tunja, otro en Duitama y un último en Sogamoso. En la Tabla 1 se presentan las conclusiones obtenidas. 
Tabla 1. Competencias emprendedores boyacenses

\begin{tabular}{|c|c|}
\hline COMPETENCIA & HALLAZGOS \\
\hline $\begin{array}{l}\text { Orientación a objetivos. Capacidad para enfocar es- } \\
\text { fuerzos en procura de alcanzar un objetivo, teniendo en } \\
\text { cuenta el costo-beneficio. (Bogotá Emprende, 2010). } \\
\text { Capacidad de encaminar los actos al logro esperado } \\
\text { (Alles, 2004). }\end{array}$ & $\begin{array}{l}\text { La mayoría de los emprendedores boyacenses posee } \\
\text { la capacidad de cumplir los objetivos que se propone, } \\
\text { confiando y aprovechando sus capacidades para } \\
\text { lograrlo. }\end{array}$ \\
\hline $\begin{array}{l}\text { Capacidad de exploración. Consiste en la acción de } \\
\text { incrementar conocimiento, sobrepasando la formula- } \\
\text { ción de preguntas relacionadas con el desarrollo nor- } \\
\text { mal de actividades y profundizar, consiguiendo mayor } \\
\text { nivel de información útil (Bogotá Emprende, 2010) }\end{array}$ & $\begin{array}{l}\text { La mayoría de los encuestados no ha desarrollado di- } \\
\text { cha capacidad, prefiere realizar las cosas de la misma } \\
\text { manera que en situaciones similares o anteriores, hay } \\
\text { baja capacidad de exploración y por lo tanto cierta } \\
\text { aversión a hacer cosas distintas o de maneras diferentes } \\
\text { o a tomar riesgos. }\end{array}$ \\
\hline $\begin{array}{l}\text { Trabajo en equipo. Capacidad para colaborar con } \\
\text { otros, formar parte de un grupo. Implica tener expec- } \\
\text { tativas positivas respecto a otros, comprenderlos y } \\
\text { generar y mantener un buen clima de trabajo (Alles, } \\
\text { 2009). Supone la capacidad de colaborar y cooperar } \\
\text { con los demás, de formar parte de un grupo y de } \\
\text { trabajar juntos (Bogotá Emprende, 2010) }\end{array}$ & $\begin{array}{l}\text { Por lo general se hace por conveniencia, ya que el } \\
\text { emprendedor boyacense tiene una baja cultura de tra- } \\
\text { bajo en equipo, hay baja tendencia a la colaboración y } \\
\text { cooperación con los demás, a menos que sea necesario } \\
\text { u obligatorio. }\end{array}$ \\
\hline $\begin{array}{l}\text { Liderazgo. Capacidad para generar compromiso y lo- } \\
\text { gar el respaldo de otros (Alles, 2009). Está dirigido por } \\
\text { un proceso de comunicación, al logro de una o varias } \\
\text { metas. Es la capacidad de autogestionar su trabajo u } \\
\text { orientar y apoyar la acción de otros al cumplimiento } \\
\text { de objetivos (Bogotá Emprende, 2010) }\end{array}$ & $\begin{array}{l}\text { La dificultad es notoria, los emprendedores boya- } \\
\text { censes sienten que poseen una baja influencia inter- } \\
\text { personal que es importante para gestionar su trabajo, } \\
\text { orientar y apoyar la acción de otros al cumplimiento } \\
\text { de los objetivos. Hay dificultad en cuanto a la co- } \\
\text { municación y el trabajo conjunto con personas que } \\
\text { puedan tener puntos de vista distintos, situación que } \\
\text { hace presumir un alto individualismo en el boyacense, } \\
\text { lo que dificulta su capacidad de liderar procesos o } \\
\text { iniciativas donde sin duda se requiere la participación } \\
\text { de múltiples personas, que comparten conocimientos } \\
\text { y expectativas diversas. }\end{array}$ \\
\hline $\begin{array}{l}\text { Proactividad. Actitud permanente de adelantarse a los } \\
\text { demás en su accionar. Es la predisposición a actuar } \\
\text { en forma proactiva y no solo pensar en qué hay que } \\
\text { hacer en el futuro (Alles, 2004). Anticiparse a las } \\
\text { situaciones, necesidades y problemas para proveer } \\
\text { soluciones (Bogotá Emprende, 2010). }\end{array}$ & $\begin{array}{l}\text { La tendencia es la de plegarse a lo que ya existe a } \\
\text { lo que ya está probado, lo que es congruente con la } \\
\text { limitada competencia en exploración. No se evidencia } \\
\text { una actitud permanente de adelantarse a los demás. }\end{array}$ \\
\hline $\begin{array}{l}\text { Pensamiento estratégico. Capacidad para compren- } \\
\text { der los cambios del entorno y establecer su impacto } \\
\text { a corto, medio y largo plazo, optimizar fortalezas y } \\
\text { actuar sobre debilidades y aprovechar oportunidades } \\
\text { (Alles, 2009). Conoce cuál es su negocio, a dónde se } \\
\text { quiere llegar y cómo alcanzar el objetivo. Hace análisis } \\
\text { estratégico (amenazas y oportunidades) para la toma } \\
\text { de decisiones (Bogotá Emprende, 2010). }\end{array}$ & $\begin{array}{l}\text { Baja capacidad de planificación, al parecer el empren- } \\
\text { dedor vive el día a día, tiene dificultades en conocer } \\
\text { cuál debe ser su negocio, orientándose solamente al } \\
\text { producto o servicio sin observar, la visión es escasa } \\
\text { para la mayoría, por lo tanto se infiere que en su } \\
\text { mayoría son negocios de subsistencia. Hay falencias } \\
\text { en el establecimiento de objetivos y por su puesto en } \\
\text { las maneras de alcanzarlos, y hay un bajo escaneo del } \\
\text { entorno. Los emprendedores presentan gran dificultad } \\
\text { en determinar metas, prioridades, acciones, plazos y } \\
\text { recursos en cuanto a su vida personal y por supuesto } \\
\text { esto se refleja también iniciativas empresariales. }\end{array}$ \\
\hline
\end{tabular}

Fuente: elaboración de los autores a partir de competencias seleccionadas. 
También se averiguó lo concerniente a la comprensión de conceptos empresariales básicos en finanzas y mercados, teniendo en cuenta que su conocimiento y aplicación se consideran importantes para el desarrollo de iniciativas empresariales pues permiten operar de manera exitosa la empresa. Para ese propósito se seleccionaron cuatro áreas: financiera, mercados, procesos estratégicos y operativos.
Fueron diseñadas cuatro preguntas para cada área, cuyo objetivo fue determinar fortalezas y debilidades que poseen los emprendedores boyacenses, lo que le permite a las entidades que promueven el emprendimiento acercarse a los conocimientos que deberían adquirir los emprendedores. En la Tabla 2 se presentan los resultados obtenidos.

Tabla 2. Conocimiento conceptos empresariales, emprendedores boyacenses

\begin{tabular}{|l|l|}
\hline \multicolumn{1}{|c|}{ Área } & \multicolumn{1}{|c|}{ Hallazgos } \\
\hline $\begin{array}{l}\text { Financiera. Se indagó acerca de conceptos financie- } \\
\text { ros básicos y estados financieros básicos, presupues- } \\
\text { tos, costos y rentabilidad. }\end{array}$ & $\begin{array}{l}\text { La mayoría (alrededor del 80\%) manifiesta conocer la } \\
\text { importancia de los estados financieros, sin embargo so- } \\
\text { lamente el 60\% dice tener algún conocimiento básico } \\
\text { acerca de los mismos. La mayoría (alrededor del 85 } \\
\text { \%) dice tener conocimiento acerca de presupuestos de } \\
\text { inversión, costos y la manera de calcular rentabilidad. }\end{array}$ \\
\hline $\begin{array}{l}\text { Mercados. Se indagó acerca de conocimiento de la } \\
\text { competencia, necesidades de clientes y estrategias de } \\
\text { mercadeo. Canales de distribución. }\end{array}$ & $\begin{array}{l}\text { En elárea de mercados se evidenció dificultad en cuanto } \\
\text { al estudio de las verdaderas necesidades del mercado, } \\
\text { los emprendedores no realizan estudios sobre el produc- } \\
\text { to y la competencia, desconocen el entorno y mercado } \\
\text { en que quieren incursionar. También hay falencias en } \\
\text { cuanto a la utilización de estrategias para alcanzar el } \\
\text { objetivo propuesto, sin un estudio de mercados no se } \\
\text { pueden establecer las estrategias por seguir para alcan- } \\
\text { zar los objetivos, siendo estas la base para que la idea } \\
\text { o modelo de negocio se desarrolle de manera exitosa. } \\
\text { Los emprendedores boyacenses, por lo general, no } \\
\text { conocen las diferentes formas o canales de distribución } \\
\text { de un producto, lo que les impide implementar planes } \\
\text { de acción que permitan llegar de manera exacta al } \\
\text { mercado objetivo. }\end{array}$ \\
\hline
\end{tabular}

Fuente: elaboración de los autores.

SEGUNDO CONCURSO DEPARTAMENTAL DE EMPRENDEDORES

El Global Entrepreneurship Monitor -GEM-(2012) reconoce que la creación de empresas es uno de los motores más importantes para el crecimiento económico de los países. En Colombia este crecimiento económico se basa en la innovación, asociado a factores como el emprendimiento, la financiación, programas gubernamentales de apoyo al emprendimiento, acompañamiento en la creación de empresas y una norma 
legal que soporte la actividad; factores enfocados hacia el aprovechamiento de oportunidades existentes en el mercado, a fin de provocar un impacto importante en la producción, el empleo y la innovación tecnológica.

La alianza entre el gobierno, la academia y el sector privado, posibilitó la identificación de ideas de negocio y nuevas empresas. En Boyacá, el proceso de fortalecimiento de las competencias emprendedoras y empresariales está enmarcado en la política nacional de emprendimiento. Esta política especifica el papel del Estado como actor dentro de los procesos emprendedores y determina el marco conceptual, jurídico e institucional que abarca el emprendimiento como responsabilidad pública. (Cepal, 2011)

En el ámbito legislativo, el proceso se sustenta en la Ley 1014 de 2006 "De fomento a la cultura del emprendimiento", cuyo objetivo es: promover el espíritu emprendedor articulando el sistema educativo y sistema productivo nacional, en el cual se trabaje conjuntamente sobre los principios que establece la constitución encaminados a fortalecer los procesos empresariales que contribuyan al desarrollo local, regional y territorial. En esta ley se establece la necesidad de articulación entre la empresa, la academia y el Estado, para fomentar y fortalecer el emprendimiento de calidad.

Sin duda alguna, el emprendimiento se ha convertido en un tema de agenda pública, académica e institucional, una de las principales razones es que el emprendimiento tiene una gran influencia en la dinámica de una economía, y la evidencia internacional demuestra que los países con mayor grado de desarrollo son los que cuentan con alto grado de emprendimiento innovador. Se puede decir, entonces, que la dinámica de una economía depende de las iniciativas empresariales, por cuanto afectan la demanda agregada, la competitividad y la necesidad de innovar para ingresar en un mercado determinado. De ahí el interés en el desarrollo y fortalecimiento de competencias emprendedoras, que se evidencien en nuevos emprendimientos e ideas de negocio con oportunidad en el mercado.

Es por eso que durante el proceso de este segundo concurso departamental de emprendimiento se realizó un acompañamiento permanente a los emprendedores para fortalecer sus competencias y conseguir que ellos mismos se convirtieran en los principales agentes en la transformación de su realidad, con el enfoque acciónparticipación permite hacer más pequeñas las brechas existentes entre la teoría y la práctica, se observa la realidad para generar cambios en la práctica. (Ortiz \& Borjas, 2008) A continuación se describe cada una de las fases desarrolladas:

Inicialmente se solicitaron registros de las bases de datos de las cámaras de comercio de Duitama, Tunja y Sogamoso, además se hicieron visitas al Sena, universidades, unidades de emprendimiento y centros de 
desarrollo empresarial del departamento, para seleccionar emprendedores y empresas de creación reciente (menos de tres años), permitiendo el contacto directo con los actores identificados. Se llevaron a cabo eventos de sensibilización que fueron programados por la Secretaría de Productividad, Tic y Gestión del Conocimiento, en Tunja, Duitama y Sogamoso, se hizo una invitación formal a los emprendedores y empresarios identificados con el objetivo de promocionar el segundo concurso departamental de emprendedores. Durante estos eventos se aplicó el instrumento de recolección de la información que permitió conocer el perfil del emprendedor boyacense y su nivel de conocimientos técnicos empresariales explicados en el segundo apartado.

Durante el diseño del concurso se elaboraron los términos de referencia, diseñados por el grupo de investigación Competitividad y Desarrollo Local -Codel-, documento que contiene la descripción y objeto del concurso, definiciones (ley y política nacional de emprendimiento), inscripción, categorías, requisitos, condiciones, modalidades de presentación, población, objetivo del proceso, reconocimientos, derechos de autor, entre otros, y se conformaron los lineamientos por seguir para la ejecución del concurso. También se elaboraron tres formatos para la inscripción de los participantes al concurso, de acuerdo con las categorías establecidas por el grupo de investigación Codel, las cuales se mencionan a continuación:

Tengo una idea: emprendedor o grupo de emprendedores (personas naturales), estudiantes de básica secundaria de los grados 10 y 11 de las instituciones educativas del departamento de Boyacá, que tienen una idea de negocio y que no han iniciado ningún proceso formal empresarial.

Quiero mi empresa: emprendedor(es) o empresario(s) que tiene un modelo de negocio en etapa de diseño o en etapa inicial de puesta en marcha.

Soy emprendedor: empresarios (personas naturales o jurídicas) que ya están consolidados, pero que no hayan superado dos años de formalización ante la entidad encargada.

Cada uno de los formatos contenía los datos personales del equipo emprendedor y la descripción de la idea o modelo de negocio, y como anexo se elaboró un instructivo para cada formato, el cual describía la forma en que el participante debía diligenciar el formato; el referente para la elaboración de los formatos fue el modelo Canvas.

La inscripción al concurso se realizó a través de la página web de la Universidad Pedagógica y Tecnológica de Colombia, espacio en que se encontró la información general, etapas, términos de referencia, 
consulta de cronograma, formatos e instructivos, proceso de inscripción y la opción de registrar dudas. Se inscribieron 76 emprendedores y empresarios. El proceso tuvo una mayor receptividad en los municipios de Tunja, Duitama y Sogamoso, en los cuales hubo mayor participación en el concurso con las ideas y modelos de negocio, probablemente porque en estos municipios se realizaron los eventos de sensibilización.

Los participantes debían llenar y subir el formato a la página web, también se solicitó que hicieran un vídeo, en el cual debían explicar su idea, para subirlo igualmente a la web. Durante esta etapa hubo acompañamiento permanente para la solución e inquietudes de quienes se inscribieron al concurso. En la Tabla 3 se presenta la participación por municipio.

En cuanto a los sectores económicos, la mayor participación se obtuvo en servicios, industria y agroindustria. Otros sectores en los que la participación es baja y que se pueden fortalecer son TIC y turismo, ya que el departamento está apostando por su desarrollo.

Tabla 3. Participación por municipio

\begin{tabular}{|c|c|}
\hline MUNICIPIO & NÚMERO \\
\hline Tunja & 33 \\
Duitama & 12 \\
Sogamoso & 13 \\
Toca & 2 \\
San Eduardo & 2 \\
Socotá & 1 \\
Garagoa & 1 \\
Labranzagrande & 1 \\
Pauna & 1 \\
Tota & 1 \\
Tópaga & 1 \\
Tinjacá & 1 \\
Gámeza & 1 \\
Paipa & 1 \\
Firavitoba & 1 \\
Miraflores & 1 \\
Nobsa & 2 \\
Soracá & 1 \\
TOTAL & $\mathbf{7 6}$ \\
\hline
\end{tabular}

Fuente: elaboración de los autores. 
Tabla 4. Participación por sectores

\begin{tabular}{|l|c|c|}
\hline \multicolumn{1}{|c|}{ SECTOR } & NÚMERO & PORCENTAJE \\
\hline Agroindustrial & 12 & 16 \\
Agropecuario & 6 & 8 \\
Artesanal & 5 & 7 \\
Comercio & 7 & 9 \\
Educación & 4 & 5 \\
Industria & 17 & 22 \\
Servicios & 20 & 26 \\
TIC 2 & 3 & 4 \\
Turismo & 3 & $\mathbf{1 0 0}$ \\
TOTAL & $\mathbf{7 6}$ & \\
\hline
\end{tabular}

Fuente: elaboración de los autores.

Tabla 5. Participación por categorías

\begin{tabular}{|l|c|c|}
\hline CATEGORÍA & NÚMERO DE PROYECTOS & PORCENTAJE \\
\hline Categoría 1. Idea de negocio & 18 & 24 \\
Categoría 2. Quiero mi empresa & 37 & 49 \\
Categoría 3. Soy emprendedor & 12 & 16 \\
No cumplen términos de referencia & 9 & 12 \\
TOTAL & $\mathbf{7 6}$ & $\mathbf{1 0 0}$ \\
\hline
\end{tabular}

Fuente: elaboración de los autores.

Durante la etapa de sensibilización se hizo evidente que para próximas oportunidades, es necesario cubrir todo el departamento, pues la mencionada sensibilización se desarrolló únicamente en los municipios más grandes (Tunja, Duitama y Sogamoso), aunque se convocó a la mayoría de los 123 municipios a través de información a alcaldías e instituciones educativas.

Una vez definido el cierre de las inscripciones, se hizo una preselección, se verificó el cumplimiento de requisitos según los términos de referencia. Quedaron 67 proyectos preseleccionados que fueron remitidos al equipo evaluador del concurso, el cual estaba conformado por diez miembros de la Red Regional de Emprendimiento, expertos en las diferentes actividades económicas, en las cuales se enmarcaban los proyectos.

Para la evaluación, se diseñó un documento con los criterios y la metodología para determinar las mejores ideas y modelos de negocio, de acuerdo con los términos de referencia. Los criterios para selección de las ideas de negocio se presentan en la Tabla 6, y los criterios para selección de modelos de negocio en la Tabla 7. Los proyectos fueron asignados teniendo en cuenta la naturaleza de los proyectos y el perfil de los evaluadores a quienes se entregaron los proyectos y los criterios de evaluación, además firmaron acuerdo de confidencialidad. Todo el proceso estuvo acompañado por el Fondo Regional de Garantías. 
Tabla 6. Criterios selección ideas de negocio

\begin{tabular}{|c|c|}
\hline CRITERIO & DESCRIPCIÓN \\
\hline Creatividad e innovación & $\begin{array}{l}\text { El equipo emprendedor presenta una solución original, } \\
\text { es nueva o contiene nuevas asociaciones entre ideas } \\
\text { ya conocidas. Tiene potencial para ser aplicada } \\
\text { exitosamente en el mercado específico al que hace } \\
\text { referencia la propuesta. }\end{array}$ \\
\hline Conocimiento técnico & $\begin{array}{l}\text { El equipo emprendedor muestra conocimiento técnico y } \\
\text { presenta con claridad su idea. }\end{array}$ \\
\hline Diferenciación & $\begin{array}{l}\text { El producto / servicio posee factores de diferenciación } \\
\text { frente a su competencia en cuanto a características } \\
\text { físicas, diseño, rendimiento, estructura de precios, canales } \\
\text { de distribución, publicidad. }\end{array}$ \\
\hline Potencial de negocio & $\begin{array}{l}\text { Hay claridad sobre los insumos que requiere el producto } \\
\text { o servicio } \\
\text { * Ha identificado los competidores, su ubicación, tamaño, } \\
\text { participación en el mercado, precio. } \\
\text { * Ha identificado productos o servicios sustitutos. } \\
\text { * Identifica a sus clientes y el nicho de mercado al que } \\
\text { pertenecen. }\end{array}$ \\
\hline Potencial de la idea en diversos mercados & $\begin{array}{l}\text { El producto/servicio cuenta con espacio para crecimiento } \\
\text { en el mercado internacional y es posible su ampliación } \\
\text { en el tiempo (crecimiento futuro) y espacio (diversos } \\
\text { países). }\end{array}$ \\
\hline $\begin{array}{l}\text { Vinculación con actividades de investigación } \\
\text { y alianzas estratégicas }\end{array}$ & $\begin{array}{l}\text { Para la realización del proyecto se contemplan alianzas } \\
\text { con instituciones que facilitan la consecución de los } \\
\text { objetivos (universidades, empresa, estado, sociedad y } \\
\text { redes). }\end{array}$ \\
\hline $\begin{array}{l}\text { Alineación con planes de gobierno y sectores } \\
\text { productivos del departamento de Boyacá }\end{array}$ & $\begin{array}{l}\text { La idea de negocio tiene alta relación con los sectores } \\
\text { sobresalientes o potenciales del departamento de Boyacá. }\end{array}$ \\
\hline $\begin{array}{l}\text { Claridad en la presentación de la idea de } \\
\text { negocio }\end{array}$ & $\begin{array}{l}\text { La presentación de la idea de negocio es clara y coherente. } \\
\text { A través del documento de presentación y el vídeo se } \\
\text { logra comprender la idea. }\end{array}$ \\
\hline
\end{tabular}

Fuente: elaboración de los autores. 
Tabla 7. Criterios selección modelo de negocio

\begin{tabular}{|c|c|}
\hline CRITERIO & DESCRIPCIÓN \\
\hline Perfil del equipo emprendedor & $\begin{array}{l}\text { El proyecto es presentado por un equipo multidisciplinario, } \\
\text { cuyos conocimientos y competencias son congruentes con } \\
\text { la naturaleza del modelo de negocio. El equipo cuenta con } \\
\text { capacidades y experiencia que sumadas contribuyen de manera } \\
\text { importante al éxito del proyecto. }\end{array}$ \\
\hline $\begin{array}{l}\text { Conocimiento técnico del equipo } \\
\text { emprendedor }\end{array}$ & $\begin{array}{l}\text { El equipo emprendedor muestra conocimiento técnico de la } \\
\text { industria en la que se establece el proyecto. }\end{array}$ \\
\hline Viabilidad técnica & $\begin{array}{l}\text { El proyecto es viable técnicamente. Es posible llevarlo a cabo } \\
\text { en las condiciones del equipo y con la tecnología disponible. }\end{array}$ \\
\hline $\begin{array}{l}\text { Uso y aplicación responsable y } \\
\text { sostenible de la tecnología }\end{array}$ & $\begin{array}{l}\text { El proyecto involucra el uso o aplicación de tecnologías limpias } \\
\text { que reducen o previenen la contaminación y el consumo de } \\
\text { recursos. }\end{array}$ \\
\hline Creatividad e innovación & $\begin{array}{l}\text { El equipo emprendedor presenta una solución original, es } \\
\text { nueva o contiene nuevas asociaciones entre ideas ya conocidas. } \\
\text { Tiene potencial para ser aplicada exitosamente en el mercado } \\
\text { específico al que hace referencia la propuesta. }\end{array}$ \\
\hline Diferenciación & $\begin{array}{l}\text { El producto / servicio posee factores de diferenciación frente } \\
\text { a su competencia en cuanto a características físicas, diseño, } \\
\text { rendimiento, estructura de precios, canales de distribución, } \\
\text { publicidad. }\end{array}$ \\
\hline Potencial de negocio & $\begin{array}{l}\text { Hay claridad sobre los insumos que requiere el producto o servicio } \\
\text { * Ha identificado los competidores, su ubicación, tamaño, } \\
\text { participación en el mercado, precio. } \\
\text { * Ha identificado productos o servicios sustitutos. } \\
\text { * Identifica a sus clientes y el nicho de mercado al que pertenecen. }\end{array}$ \\
\hline $\begin{array}{l}\text { Potencial de la idea en diversos } \\
\text { mercados }\end{array}$ & $\begin{array}{l}\text { El producto/servicio cuenta con espacio para crecimiento en el } \\
\text { mercado internacional y es posible su ampliación en el tiempo } \\
\text { (crecimiento futuro) y espacio (diversos países). }\end{array}$ \\
\hline Oportunidad de inversión & $\begin{array}{l}\text { La propuesta presenta una oportunidad de inversión para } \\
\text { empresas o personas con recursos. }\end{array}$ \\
\hline $\begin{array}{l}\text { Potencial de la idea en diversos } \\
\text { mercados }\end{array}$ & $\begin{array}{l}\text { El producto / servicio cuenta con espacio para crecimiento en el } \\
\text { mercado internacional y es posible su ampliación en el tiempo } \\
\text { (crecimiento futuro) y espacio (diversos países). }\end{array}$ \\
\hline $\begin{array}{l}\text { Potencial de impacto económico } \\
\text { social y ambiental }\end{array}$ & $\begin{array}{l}\text { Potencial de impacto económico social y ambiental. El desarrollo } \\
\text { o mejoramiento del proyecto es importante para el desarrollo } \\
\text { económico, social y ambiental. }\end{array}$ \\
\hline $\begin{array}{l}\text { Vinculación con actividades de } \\
\text { investigación y alianzas estratégicas }\end{array}$ & $\begin{array}{l}\text { Para la realización del proyecto se contemplan alianzas con } \\
\text { instituciones que facilitan la consecución de los objetivos } \\
\text { (universidades, empresa, Estado, sociedad y redes). }\end{array}$ \\
\hline $\begin{array}{l}\text { Alineación con planes de gobierno } \\
\text { y sectores productivos del } \\
\text { departamento de Boyacá }\end{array}$ & $\begin{array}{l}\text { La idea de negocio tiene alta relación con los sectores } \\
\text { sobresalientes o potenciales del departamento de Boyacá. }\end{array}$ \\
\hline $\begin{array}{l}\text { Claridad en la presentación de la } \\
\text { idea de negocio }\end{array}$ & $\begin{array}{l}\text { La presentación de la idea de negocio es clara y coherente. } \\
\text { A través del documento de presentación y el vídeo se logra } \\
\text { comprender la idea. }\end{array}$ \\
\hline
\end{tabular}

Fuente: elaboración de los autores. 
Durante esta primera ronda de evaluación, se evidenció que difícilmente las ideas y modelos de negocio satisfacían los criterios establecidos, es más, ninguna de las propuestas los satisfizo en un 50 $\%$, con lo cual se puede concluir que el emprendedor el departamento requiere mayor capacitación, asesoría y acompañamiento en cuanto a conocimientos básicos empresariales y planes de negocio. También hace pensar que las instituciones educativas del departamento deben mejorar la formación en emprendimiento brindando a los estudiantes no solo conceptos sino también herramientas de análisis útiles para toma de decisiones empresariales (financieras, de mercados).

Como resultado de esta primera evaluación se seleccionaron diez ideas de negocio como ganadoras del reconocimiento de la categoría uno (1); once modelos de negocio de categoría dos (2) y ocho modelos de negocio de categoría tres (3). Los resultados se presentan en las tablas 8,9 y 10.

Tabla 8. Finalistas categoría 1. Tengo una idea.

\begin{tabular}{|c|l|c|c|}
\hline No & \multicolumn{1}{|c|}{ NOMBRE } & CIUDAD & SECTOR \\
\hline 1 & Fábrica La Cascarita & Tunja & Agroindustria \\
\hline 2 & Guía turística & Tunja & Turismo \\
\hline 3 & Microideas & Duitama & Industria \\
\hline 4 & Postres y ponqués & Tunja & Industria \\
\hline 5 & $\begin{array}{l}\text { Uso de la yuca, bore, chachafruto y } \\
\text { caña de azúcar }\end{array}$ & San Eduardo & Agroindustria \\
\hline 6 & Horno tipo cámara & Tunja & Industria \\
\hline 7 & Cerámica & Tunja & Comercio \\
\hline 8 & Productos Libasart & Tunja & Agroindustria \\
\hline 9 & Comida italiana & Tunja & Comercio \\
\hline 10 & Postres & Tunja & Agroindustria \\
\hline
\end{tabular}

Fuente: elaboración de los autores.

Tabla 9. Finalistas categoría 2. Quiero mi empresa.

\begin{tabular}{|c|l|c|c|}
\hline No & \multicolumn{1}{|c|}{ NOMBRE } & CIUDAD & SECTOR \\
\hline 1 & Camino muisca & Sogamoso & Turismo \\
\hline 2 & Arte y cultura gastronómica & Tunja & Turismo e industria \\
\hline 3 & Centro ecoturismo & Sogamoso & Turismo \\
\hline 4 & Café Macana & Sogamoso & Agropecuario \\
\hline 5 & Aplicaciones para decorar viviendas & Duitama & Industria \\
\hline 6 & $\begin{array}{l}\text { 200 señas técnicas para ensamble y } \\
\text { mantenimiento de redes y computadores. }\end{array}$ & Tunja & Servicios \\
\hline 7 & Forrajes verde & Tunja & Agropecuario \\
\hline 8 & Todo a mano & Sogamoso & Artesanal \\
\hline 9 & Las delicias de mi tierra & Tota & Agroindustria \\
\hline 10 & Talleres carpintería porvenir & San Eduardo & Industria \\
\hline 11 & Centro terapéutico muisca & Tunja & Servicios \\
\hline
\end{tabular}

Fuente: elaboración de los autores. 
Las competencias emprendedoras en el departamento de Boyacá

Diana Cristina Rodríguez Moreno - Adriana Ximena Gómez Murillo

Tabla 10. Finalistas categoría 3. Soy emprendedor.

\begin{tabular}{|c|l|c|c|}
\hline No & \multicolumn{1}{|c|}{ NOMBRE } & CIUDAD & SECTOR \\
\hline 1 & Soluciones profesionales Group SAS & Sogamoso & Servicios \\
\hline 2 & Quinua de los Andes & Soracá & Industria \\
\hline 3 & $\begin{array}{l}\text { Limpieza y embellecimiento de } \\
\text { tractocamiones }\end{array}$ & Duitama & Servicios \\
\hline 4 & Editic & Tunja & Educación \\
\hline 5 & Ecosistem - Diseño publicitario & Tunja & Servicios \\
\hline 6 & $\begin{array}{l}\text { Técnicas y estrategias en } \\
\text { comunicaciones SAS }\end{array}$ & Miraflores & Agropecuario \\
\hline 7 & $\begin{array}{l}\text { Cultivo, transformación y } \\
\text { comercialización de la chamba }\end{array}$ & Tunja & Comercio \\
\hline 8 & Pomarosa café gourmet & &
\end{tabular}

Fuente: elaboración de los autores.

Se hizo una segunda ronda de evaluación, en que el método utilizado fue el panel de expertos. Para la etapa final, cada uno de los equipos seleccionados en la primera ronda (tablas 8,9 y 10) debió presentarse y exponer sus ideas ante el panel de jurados. Para esta etapa se establecieron metodología y criterios para evaluación, consensuados con los jurados e informados previamente a cada uno de los participantes, con la respectiva fecha, lugar y hora de presentación. Los criterios establecidos fueron muy sencillos, comparados con la primera evaluación, pues como ya se mencionó, los emprendedores están lejos de cumplir con los criterios iniciales. Estos fueron: propuesta de valor, mercados, técnico, perfil del emprendedor y financiero. Incluso con la simplificación de los criterios y disminución de exigencia, se observó que los emprendedores difícilmente comprenden conceptos económicos, financieros y empresariales y para algunos fue muy difícil la presentación de su idea ante el público.
Finalmente se otorgó reconocimiento social y económico a cada uno de los ganadores en ceremonia pública realizada el 20 de junio del 2013 en el Centro de Convenciones de la Cámara de Comercio de Tunja, la cual contó con la asistencia de funcionarios de la Secretaría de Productividad, Tic y Gestión del Conocimiento de la Gobernación de Boyacá, investigadoras de la Universidad Pedagógica y Tecnológica de Colombia del Grupo de Investigación GEVAFI, miembros de la Red Regional de Emprendimiento, los emprendedores y empresarios que participaron del proceso de fortalecimiento de las competencias emprendedoras y empresariales del departamento de Boyacá y medios de comunicación.

\section{CONCLUSIONES}

Los emprendedores boyacenses confían en su capacidad para el cumplimiento de objetivos. El desarrollo de esta competencia es de gran importancia, 
pues, de hecho, el sentirse capaz de realizar algo es fundamental para lograrlo. El emprendedor boyacense es capaz de combinar recursos disponibles para solucionas situaciones particulares.

En cuanto a la capacidad de exploración, es necesario un trabajo fuerte, pues generalmente el emprendedor boyacense tiene una limitada capacidad de exploración, lo que indudablemente limita la capacidad de innovación y, por lo tanto, de descubrir o generar ideas nuevas para la solución de problemas regionales. Esta baja capacidad de exploración probablemente está relacionada con algunos rasgos del sistema educativo, orientado a contenidos donde el estudiante cree sin dudar en todo lo que dice el maestro. Incluso en la universidad se observa una falta de deseo de explorar, conocer cosas nuevas, pues el estudiante, por lo general, va hasta donde el profesor le indica; así que es necesario despertar en lo jóvenes ese espíritu explorador necesario para el avance científico, tecnológico y empresarial. Se requiere mejorar la educación hacia el emprendimiento, no acerca del emprendimiento (Osorio \& Pereira, 2001)

El emprendedor también presenta baja capacidad de trabajo en equipo, pues prefiere, en principio, hacer las cosas solo, tal vez por la baja confianza en otras personas. Efectivamente, el pensamiento en general es que el trabajar con otros trae problemas, genera conflictos y existe el peligro de que se quieran aprovechar de sus ideas. Es necesario que el conocimiento se conjugue, como dirían Nonaka y Takeuchi (1995), que ese conocimiento tácito que hay en cada persona se comparta, se socialice con otros, este es el primer paso en esa espiral del conocimiento. También es apremiante buscar mecanismos que permitan generar confianza entre las personas, tener la certeza de que el trabajo en equipo permite beneficios mutuos, genera sinergia. Las universidades de la región necesitan mayor intra e intercomunicación, los estudiantes de las distintas facultades en cada universidad y de diferentes universidades, deben conocerse, trabajar juntos, encontrar maneras de convertir ideas de pares técnicos y tecnológicos en negocios.

Si hay baja propensión al trabajo en equipo, con un marcado individualismo, pues es de esperarse que la competencia en liderazgo también sea débil, el principio en liderazgo implica influir en otros para lograr un propósito, significa también generar una visión compartida de lo que se quiere y si es difícil trabajar con otros, comunicarse con otros, conocer sus expectativas, capacidades, etc., pues es remoto conseguir influenciarlos.

El emprendimiento necesita del pensamiento estratégico y en este aspecto el emprendedor boyacense debe construir una mayor capacidad de planificación. Si se parte de la falta de claridad en los objetivos, qué decir entonces de las estrategias para alcanzar algo que es incierto. Por otro lado, una gran cantidad de emprendedores no 
conoce de manera profunda su negocio, es probable que esta condición se deba a que son emprendimientos por necesidad y no por oportunidad y el emprendedor se encuentra conforme con recibir lo necesario para cubrir ciertas necesidades. Por otra parte, para el emprendimiento se debe generar un ecosistema adecuado y uno de los componentes fundamentales es la infraestructura, puesto que el acceso a muchos municipios es difícil, lo que genera barreras para los empresarios

El departamento debe trabajar intensamente en el fortalecimiento de competencias emprendedoras, focalizándose en los sectores que ha declarado como potenciales; hay que tener en cuenta que el hecho de apostar a todo genera desgaste y sectores débiles. Durante el proceso del segundo concurso departamental de emprendimiento, se observó el bajo conocimiento de la comunidad en conceptos básicos administrativos y económicos $\mathrm{y}$ en general en la formulación de un plan de negocio.

En un próximo concurso o actividades de fomento a la cultura emprendedora, se requiere llegar a todos los municipios, pues en esta oportunidad prácticamente solo hubo participación de Tunja, Duitama y Sogamoso. Este ejercicio permitió conocer algunas falencias en competencias y también problemas sociales que aquejan a ciertos grupos y en los cuales el gobierno departamental y las universidades deben intervenir.

Se deben identificar y fomentar de manera más decidida los emprendimientos de oportunidad, pues aunque en el concurso se presentaron algunos, la mayoría fueron por necesidad, lo que generó en los jurados del segundo concurso regional de emprendimiento, cierta compasión hacia algunos emprendedores, debido a sus difíciles condiciones de vida; razón por la que también se requiere el trabajo en innovación social y emprendimiento social. Igualmente cabe destacar el papel fundamental que tiene la universidad en el acompañamiento a los emprendedores y sus ideas, realizando seguimiento a estudios de factibilidad, planes de negocio y gestión empresarial.

Es importante reconocer que emprender no necesariamente tiene que ver con la actividad empresarial, pues emprender significa comenzar algo, una obra o un negocio, un empeño, lo que encierra dificultad o peligro (RAE). La presencia de riesgo hace que no todas las personas tomen el riesgo de emprender, así que uno de los campos de estudio del emprendimiento, es precisamente identificar cuáles son esas características, habilidades y rasgos que tiene los emprendedores. 


\section{REFERENCIAS}

Alda-Varas, R., Villardón-Gallego, L. \& Elexpuru-Albizuri, I. (2012). Propuesta y validación de un perfil de competencias de la persona emprendedora. Implicaciones para la formación. Electronic Journal of Research in Educational Psychology, 10(28), 1057-1080. Recuperado de http://www.redalyc.org/articulo.oa?id=293124654006

Alles, M. (2004). Diccionario de comportamientos. Gestión por competencias. La trilogía. Buenos Aires: Gránica.

Alles, M. (2009). Diccionario de competencias. La trilogía. Buenos Aires: Gránica.

Antonia, M., Ciencias, R. De, Ve, S., Zulia, U. \& Cervilla, M. A. (2013). Modelos de negocio de emprendimientos por y para la base de la pirámide. Revista de Ciencias sociales, XIX, 289-308.

Baptista, R., Lima, F. \& Preto, M. T. (2012). Entrepreneurial skills and workers' wages in small firms. Small Business Economics, 40(2), 309-323. Doi:10.1007/s11187-012-9463-7.

Birds, R. (2014). Entrepreneur-managers in higher education: (how) do they exist? Journal of Higher Education Policy and Management, 36(1), 62-73. doi:10.1080/136008 0X.2013.844663

Bogotá Emprende (2010). Identifica y potencializa tus competencias emprendedoras. Recuperado de http://www.bogotaemprende.com/documentos/3551_26_03_10_1doc_ competencias.pdf.

Bosma, N. et al. (2012). Entrepreneurship and role models. Journal of Economic Psychology $33(2), 410-424$.

Castellanos, D., Chávez P. \& Jiménez N. (2003). Propuesta de formación en liderazgo y emprendimiento. INNOVAR Revista de Ciencias Administrativas y Sociales, (22) 145-156. Recuperado de http://estudiosterritoriales.org/articulo.oa?id=81802214

Castellanos, O. (2006). Bases conceptuales e impacto de la implementación de ompetencias laborales en la relación individuo organización. Cuadernos de Administración, 19, 81 - 101.

Cepal (2011) Apoyando a las pymes: Políticas de fomento en América Latina y el Caribe. Recuperado http://www.cepal.org/publicaciones/xml/0/45410/lcr.2180.pdf.

D'Este, P., Mahdi, S., Neely, A. \& Rentocchini, F. (2012). Inventors and entrepreneurs in academia: What types of skills and experience matter? Technovation, 32(5), 293-303. Doi:10.1016ñ/j.technovation.2011.12.005 
Duarte, T. \& Ruiz, M. (2009). Emprendimiento, una opción para el desarrollo. Scientia Et Technica, XV(43), 326-331. Recuperado de http://www.redalyc.org/articulo. oa? $\mathrm{id}=84917310058$

Fernández, L. \& Fernández, E. (2010). Competencias emprendedoras y desarrollo del espiritu empresarial en los centros educativos. Recuperado de http://www.redalyc.org/articulo. oa?id=70618037001

Gallard, M.A. \& Jacinto, C. (1995). Competencias laborales tema clave en la articulación educación-trabajo. En CIID-CENEP. Boletín de la Red Latinoamericana de Educación y Trabajo, No.2. Buenos Aires, Argentina: mimeo.

Gómez, M. \& Satizábal, K. (2011). Educación en emprendimiento: fortalecimiento de competencias emprendedoras en la Pontificia Universidad Javeriana Cali. Economía, Gestión y Desarrollo, (111), 121-155.

GEM. (2012). Global Entrepreneurship Monitor - Colombia 2011 - 2012. Recuperado de http://www.gemconsortium.org/docs/2820/gem-colombia-2012-report.

Heinonen, S. \& Ruotsalainen, J. (2012). Toward the Age of Neo-Entrepreneurs. World Future Review, 4(2), 123-133. Doi:10.1177/194675671200400216

Higuita, D., Molano, J. \& Rodríguez, M. (2011). Competencias necesarias en los grupos de investigación de la Universidad Nacional de Colombia que generan desarrollos de base tecnológica. INNOVAR Revista de Ciencias Administrativas y Sociales, 21(41) 209-224. Recuperado de http://www.redalyc.org/articulo.oa?id=81822806016

Karp, T. (2006). The inner entrepreneur: A constructivistic view of entrepreneurial reality construction. Journal of Change Management, 6(3), 291-304. Doi: $10.1080 / 14697010600876864$

Lazear, E. P., Kreps, D., Lucas, R., Mcmillan, J., Oyer, P., \& Prendergast, C. (2005). Entrepreneurship. University of Chicago. Journal of Labor Economics, 23(4). Stanford, CA.

Ley 1014 de 2006. Recuperado de: www.mincit.gov.co

Lordkipanidze, M. Brezel, H. \& Backman, M. (2005). The entrepreneurship factor in sustainable tourism development. Journal of Cleaner Production 13(8), 787 - 798.

Martínez, F. \& Carmona, G. (2009). Aproximación al concepto de competencias emprendedoras: valor social e implicaciones educativas. REICE Revista Iberoamericana sobre Calidad, Eficacia y Cambio en Educación, 7(3) 82-98. Recuperado de http://www.redalyc.org/ articulo.oa?id=55114063007. 
Matiz, F. (2009). Investigación en emprendimiento, un reto para la construcción de conocimiento. Revista Escuela de Administración de Negocios EAN, (66), 169-182.

Mertens, L. (2000). La gestión por competencia laboral en la empresa y formación profesional. Madrid: Organización de Estados Iberoamericanos para la Educación, la Ciencia y la Cultura

Nonanka, I. \& Takeuchi, H. (1995). La organización creadora de conocimiento. Oxford: Oxford University.

O'Connor, A. (2013). A conceptual framework for entrepreneurship education policy: Meeting government and economic purposes. Journal of Business Venturing 28(4), 546-563.

Orrego, C. (2008, julio-diciembre). La dimensión humana del emprendimiento. Revista Ciencias Estratégicas, 16. Recuperado de http://www.redalyc.org/articulo.oa?id=151312829001> ISSN 1794-8347

Osorio, F. \& Pereira, F. (2011, julio-diciembre). Hacia un modelo de educación para el emprendimiento: una mirada desde la teoría social cognitiva. Cuadernos de Administración, 24. Recuperado de http://www.redalyc.org/articulo.oa?id=20521435001

Plehn-Dujowich, J. (2009). A theory of serial entrepreneurship. Small Business Economics, 35(4), 377-398. Doi:10.1007/s11187-008-9171-5

Ras, P. \& W. J. V. Vermeulen (2009). Sustainable production and the performance of South African entrepreneurs in a global supply chain. The case of South African table grape producers. Sustainable Development 17(5), 325 - 340.

Rhee, K. S. \& White, R. J. (2007). The Emotional Intelligence of Entrepreneurs. Journal of Small Business \& Entrepreneurship, 20(4), 409-425. Doi:10.1080/08276331.2007.10593408

Rodríguez, A. (2009). Nuevas perspectivas para entender el emprendimiento empresarial. Pensamiento \& Gestión, (26) 94-119. Recuperado de http://www.redalyc.org/articulo. oa? $\mathrm{id}=64612291005$

Santos, S. C., Caetano, A. \& Curral, L. (2013). Psychosocial aspects of entrepreneurial potential. Journal of Small Business \& Entrepreneurship, 26(6), 661-685. Doi:10.1080/08276331 .2014 .892313

Sambasivan, M. et al. (2009). Impact of personal qualities and management skills of entrepreneurs on venture performance in Malaysia: Opportunity recognition skills as a mediating factor. Technovation 29(11), 798-805.

Shumpeter, J. (1943). Capitalism, socialism and democracy. Routledge. London and New York. Taylor and Francis e-Library. Recuperado de: Digamo.free.fr/capisoc.pdf. 
Sobrado, F. \& Fernández, E. (2010). Competencias emprendedoras y desarrollo del espíritu empresarial en los centros educativos. Educación XX1, 13(1), 15-38. Recuperado de http:// www.redalyc.org/articulo.oa?id=70618037001

Soriano, D. R. \& Huarng, K.-H. (2013). Innovation and entrepreneurship in knowledge industries. Journal of Business Research 66(10), 1964-1969.

Stuetzer, M., Goethner, M. \& Cantner, U. (2012). Do balanced skills help nascent entrepreneurs to make progress in the venture creation process? Economics Letters, 117(1), 186-188. Doi:10.1016/j.econlet.2012.05.002

Stuetzer, M., Obschonka, M., Davidsson, P. \& Schmitt-Rodermund, E. (2013). Where do entrepreneurial skills come from? Applied Economics Letters, 20(12), 1183-1186. Doi:1 $0.1080 / 13504851.2013 .797554$

Stuetzer, M., Obschonka, M. \& Schmitt-Rodermund, E. (2013). Balanced skills among nascent entrepreneurs. Applied Economics Letters, 93-114. Doi:10.1007/s11187-012-9423-2

Tocher, N., Oswald, S. L. \& Shook, C. L. (2010, May). Extending the social competence perspective. Entrepreneurship \& Regional Development, 37-41. Doi:10.1080/0898562 6.2010 .535856

Zapalska, A. M., Dabb, H. \& Perry, G. (2003). Environmental Factors Affecting Entrepreneurial Activities: Indigenous Maori Entrepreneurs of New Zealand. Asia Pacific Business Review, 10(2), 160-177. Doi:10.1080/13602380410001677191 